\title{
Relativistic Iron Emission Lines in Neutron Star Low\#Mass X\#Ray Binaries as Probes of Neutron Star Radii
}

\section{Citation}

Cackett, Edward M., Jon M. Miller, Sudip Bhattacharyya, Jonathan E. Grindlay, Jeroen Homan, Michiel van der Klis, M. Coleman Miller, Tod E. Strohmayer, and Rudy Wijnands. 2008.

"Relativistic Iron Emission Lines in Neutron Star Low\#Mass X\#Ray Binaries as Probes of Neutron Star Radii." The Astrophysical Journal 674 (1): 415-20. https://doi.org/10.1086/524936.

\section{Permanent link}

http://nrs.harvard.edu/urn-3:HUL.InstRepos:41399731

\section{Terms of Use}

This article was downloaded from Harvard University's DASH repository, and is made available under the terms and conditions applicable to Other Posted Material, as set forth at http:// nrs.harvard.edu/urn-3:HUL.InstRepos:dash.current.terms-of-use\#LAA

\section{Share Your Story}

The Harvard community has made this article openly available.

Please share how this access benefits you. Submit a story.

\section{Accessibility}




\title{
RELATIVISTIC IRON EMISSION LINES IN NEUTRON STAR LOW-MASS X-RAY BINARIES AS PROBES OF NEUTRON STAR RADII
}

\author{
Edward M. Cackett, ${ }^{1,2}$ Jon M. Miller, ${ }^{1}$ Sudip Bhattacharyya, ${ }^{3,4}$ Jonathan E. Grindlay, ${ }^{5}$ Jeroen Homan, ${ }^{6}$ \\ Michiel van der Klis, ${ }^{7}$ M. Coleman Miller, ${ }^{4}$ Tod E. Strohmayer, ${ }^{8}$ and Rudy Wijnands ${ }^{7}$ \\ Received 2007 August 13; accepted 2007 October 26
}

\begin{abstract}
Using Suzaku observations of three neutron star low-mass X-ray binaries (Ser X-1, 4U 1820-30, and GX 349+2) we have found broad, asymmetric, relativistic Fe K emission lines in all three objects. These Fe K lines can be well fit by a model for lines from a relativistic accretion disk ("diskline"), allowing a measurement of the inner radius of the accretion disk and hence an upper limit on the neutron star radius. These upper limits correspond to $14.5-16.5 \mathrm{~km}$ for a 1.4 $M_{\odot}$ neutron star. The inner disk radii that we measure with Fe $\mathrm{K}$ lines are in good agreement with the inner disk radii implied by $\mathrm{kHz}$ QPOs observed in both 4U 1820-30 and GX 349+2, supporting the inner disk origin for $\mathrm{kHz}$ QPOs. In addition, the Fe $\mathrm{K}$ lines observed in these neutron stars are narrower than those in the black holes that are thought to be close to maximally spinning, as one would expect if inferences for spin are robust.
\end{abstract}

Subject headings: accretion, accretion disks — stars: neutron — X-rays: binaries

\section{INTRODUCTION}

The equation of state of the ultradense matter in neutron stars remains enigmatic; however, it is through accurate measurements of neutron star radii and masses that it can be constrained (e.g., Lattimer \& Prakash 2007; Klähn et al. 2006). Unfortunately, there are very few accessible and robust observational measurements of neutron star radii.

There are currently several methods that utilize X-ray spectroscopy/timing to determine neutron star radii. For instance, if one takes the origin of the $\mathrm{kHz}$ quasi-periodic oscillations (QPOs) to be associated with the inner edge of the accretion disk, then this places constraints on mass and radius (Miller et al. 1998). However, while these $\mathrm{kHz}$ QPOs can be measured precisely and are likely associated with the inner accretion disk, their origin is not certain (Lamb \& Miller 2001; Stella et al. 1999; Abramowicz et al. 2003; Titarchuk 2002). Burst oscillations during thermonuclear X-ray bursts may be used to constrain the neutron star radius (Bhattacharyya et al. 2005), but this method alone cannot provide very tight constraints, as there are many unknown parameters. Alternatively, thermal continuum emission from the stellar surface can give a measure of radius (e.g., van Paradijs \& Lewin 1987; Heinke et al. 2006; Zavlin 2008; Ho et al. 2007; Bogdanov et al. 2007) but suffers from distance and/or model uncertainties. In addition, X-ray absorption lines from the stellar surface would measure the gravitational redshift, but these lines appear to be extremely rare and difficult to observe, and the only detection is of modest statistical significance (Cottam et al. 2002;

\footnotetext{
1 Department of Astronomy, University of Michigan, 500 Church Street, Ann Arbor, MI 48109-1042; ecackett@umich.edu.

2 Dean McLaughlin Postdoctoral Fellow.

3 CRESST and Astrophysics Science Division, NASA/Goddard Space Flight Center, Greenbelt, MD 20771.

4 Department of Astronomy, University of Maryland, College Park, MD 20742-2421.

5 Harvard-Smithsonian Center for Astrophysics, 60 Garden Street, Cambridge, MA 02138.

${ }^{6}$ MIT Kavli Institute for Astrophysics and Space Research, 70 Vassar Street, Cambridge, MA, 02139.

7 Astronomical Institute "Anton Pannekoek," University of Amsterdam, Kruislaan 403, 1098 SJ, Amsterdam, the Netherlands.

8 Astrophysics Science Division, NASA/GSFC, Greenbelt, MD 20771.
}

Kong et al. 2007). In this paper we present a method for placing upper limits on neutron star radii using relativistic iron emission lines (see Miller 2007 for a review) that originate in the inner accretion disks around the neutron stars in X-ray binaries.

Velocity shifts encoded into disk emission lines directly reflect the radius at which the line is formed. In the X-ray spectra of accreting black holes and neutron stars, iron $\mathrm{K}$ shell emission lines are widely observed (Nandra et al. 1997; Reynolds 1997; White et al. 1985, 1986; Hirano et al. 1987; Asai et al. 2000). The extreme red wing of some iron emission lines implies that they are formed in the inner accretion disks and are primarily shaped by dynamics including relativistic Doppler shifts, due to the high velocities in the disk, and gravitational redshifts (hence, the lines are referred to as being "relativistic"). Therefore, iron K lines can serve as incisive measures of the inner disk radius (Fabian et al. 1989).

These relativistic, asymmetric $\mathrm{Fe} \mathrm{K}$ emission lines are well known and well studied in the X-ray spectra of both supermassive black holes in active galactic nuclei (Nandra et al. 1997; Reynolds 1997) and stellar-mass black holes in X-ray binaries (Miller et al. 2004). In the case of neutron stars, iron emission lines are weaker, however, and until lately prior observations have not clearly revealed a relativistic line profile, and the lines could be well fit by just a Gaussian (e.g., White et al. 1986; Asai et al. 2000; Oosterbroek et al. 2001; Di Salvo et al. 2005). Only very recently have observations of Serpens X-1 with XMM-Newton shown a relativistic, asymmetric line in a neutron star low-mass X-ray binary (LMXB) for the first time (Bhattacharyya \& Strohmayer 2007), confirming the inner disk nature of the lines in neutron star LMXBs. We note that although the iron line in Serpens X-1 has been observed before (White et al. 1986; Asai et al. 2000; Oosterbroek et al. 2001), the new observations by Bhattacharyya \& Strohmayer (2007) were the first time a clear asymmetry has been seen in the line profile.

The high effective area of the Suzaku X-ray telescope (Mitsuda et al. 2007) in the Fe K band, combined with its broadband energy coverage, means that it is optimized for studies of inner disks and relativistic regimes using iron lines. Here we present Suzaku observations of the Fe $\mathrm{K}$ lines in three neutron star LMXBs (Serpens $\mathrm{X}-1,4 \mathrm{U} 1820-30$, and GX 349+2). We find them all to show a relativistic asymmetric profile. In $\S 2$ we describe the data analysis 
TABLE 1

Summary of SUZAKU ObSERVATIONS

\begin{tabular}{cccc}
\hline \hline & & \multicolumn{2}{c}{ Exposure Time of Spectrum (ks) } \\
\cline { 3 - 4 } ObJect & Observation Date & XIS & PIN \\
\hline Serpens X-1 ......... & 2006 Oct 24 & 30.5 & 27.5 \\
4U 1820-30 ......... & 2006 Sep 14 & 17.9 & 28.4 \\
GX 349+2 ............ & 2006 Mar 14 & 25.3 & 17.3 \\
& 2006 Mar 19 & 28.1 & 22.9 \\
\hline
\end{tabular}

and results, and in $\S 3$ we discuss the use of these lines as probes of neutron star radii, as well as comparing the results with $\mathrm{kHz}$ QPOs seen in these sources and with iron lines in black holes. Finally, we summarize our findings in $\S 4$.

\section{DATA ANALYSIS AND RESULTS}

The data were obtained with the Suzaku X-ray telescope, which has several detectors on board. The X-ray Imaging Spectrometer (XIS) detectors provide spectra from 0.5 to $10 \mathrm{keV}$, while the Hard X-ray Detector (HXD) PIN camera provides spectra at higher energies from 12 to $40 \mathrm{keV}$ (with current calibrations). For observations of Serpens X-1 and 4U 1820-30, Suzaku was operated at the XIS nominal pointing position, with the XIS detectors operating in $\frac{1}{4}$ window mode and $1 \mathrm{~s}$ burst clock mode to prevent pile-up. The observations of GX 349+2 were obtained from the Suzaku data archive. Two observations were performed of approximately $25 \mathrm{ks}$ each, with the telescope pointing at the HXD nominal position. The XIS was operated with $\frac{1}{8}$ window mode and $0.3 \mathrm{~s}$ burst clock mode. As the observations are only 4 days apart and the source count rate and spectral shape did not vary significantly, we add the spectra from both observations together to improve the signal-to-noise ratio. The observations are summarized in Table 1.

The standard data spectral products for the XIS were used for all observations. We also used the canned response matrices, as custom responses cannot currently be created with the burst mode. For the HXD PIN data, we extracted the spectrum from the cleaned PIN event files following the standard analysis threads. The PIN non-X-ray background was extracted from the timedependent, observation-specific model provided by the instrument team, and was combined with the standard model for the cosmic X-ray background.

We fit the spectra using XSPEC version 11 (Arnaud 1996). The continuum fit is performed from 1 to $9 \mathrm{keV}$ in the XIS and 12 to $25 \mathrm{keV}$ in the HXD PIN. We ignore below $1 \mathrm{keV}$, as this is the region where the accumulation of some carbon-rich material onto the CCDs has changed the effective area curve; additionally, we ignore the $1.5-2.5 \mathrm{keV}$ region, where there are large calibration uncertainties due to an instrumental feature and the $4-7.5 \mathrm{keV}$ region, where the broadened iron line profile is present. We only fit the PIN spectrum up to $25 \mathrm{keV}$, as this is the energy at which the background dominates over the source flux for these objects. While the XIS consists of four separate detectors, we only fit XIS 2 and XIS 3, as their calibrations appear to be most robust. The XIS 2, XIS 3, and PIN are all fit simultaneously. The broadband energy coverage provided by the combination of both the XIS and PIN allows an accurate determination of the continuum shape on either side of the Fe K band. This is vital in robustly determining the line profile.

It is well known that neutron star spectra can be fit by a wide variety of different models (e.g., Lin et al. 2007). For simplicity, we fit a model with two thermal components (multicolor disk blackbody + blackbody) as per a recent study (Lin et al. 2007). In that study as the sources vary in luminosity, the measured temperatures of both thermal components in this model are found to follow $L \propto T^{4}$, suggesting a strong physical motivation, whereas in other models the measured temperatures of the thermal component do not follow $L \propto T^{4}$ as the source luminosity increases. An additional power-law component is also required (as is also found in Lin et al. 2007), and we note here that all three spectral components are required. The spectral index of the power law is allowed to vary between the XIS 2 and XIS 3, as this is required to remove some slight differences in slope between the calibration of the different detectors. The power-law component for the PIN is tied to the XIS 2 value, and this is the value quoted in Table 2. The parameters from the continuum spectral fits for each object are given in Table 2, and the spectral fits to Ser X-1 are shown in Figure 1 (spectral fits to the other objects are similar). All uncertainties quoted throughout the paper are at the $90 \%$ confidence level.

Fitting the continuum reveals significant iron lines in all three objects, which are all detected at greater than the $7 \sigma$ level in all objects (which we determine from an F-test). We show the lines in Figure 2. Importantly, the lines in these objects are all clearly revealed as having broad, asymmetric profiles. While we tried fitting a variety of different continuum models, the model described above is the only model that successfully fits both the $\mathrm{XIS}$ and PIN data. However, various other models, such as a disk blackbody + power-law or a blackbody + power-law model, give acceptable fits to the XIS data alone. It is important to note that the iron line profile recovered from these different continuum models is robust and changes very little in shape. This demonstrates that the line is not due to continuum components crossing in the iron band.

TABLE 2

Parameters of the Continuum Models

\begin{tabular}{|c|c|c|c|}
\hline Parameter & Ser X-1 & $4 \mathrm{U} 1820-30$ & GX $349+2$ \\
\hline$N_{\mathrm{H}}\left(10^{22} \mathrm{~cm}^{-2}\right) \ldots \ldots \ldots$ & $0.56 \pm 0.01$ & $0.26 \pm 0.01$ & $0.93 \pm 0.01$ \\
\hline 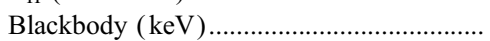 & $2.28 \pm 0.02$ & $2.46 \pm 0.03$ & $2.22 \pm 0.01$ \\
\hline Blackbody normalization $\left(10^{-2}\right) \ldots \ldots \ldots \ldots \ldots \ldots$ & $4.9 \pm 0.2$ & $3.2 \pm 0.1$ & $9.7 \pm 0.1$ \\
\hline Disk blackbody $(\mathrm{keV})$ & $1.21 \pm 0.01$ & $1.15 \pm 0.02$ & $1.29 \pm 0.01$ \\
\hline Disk blackbody normalization....................... & $103 \pm 5$ & $55 \pm 3$ & $130 \pm 5$ \\
\hline Power-law index & $3.6 \pm 0.4$ & $2.1 \pm 0.1$ & $1.4 \pm 0.1$ \\
\hline Power-law norm $\left(10^{-2}\right) \ldots \ldots \ldots \ldots$ & $18 \pm 3$ & $8.17 \pm 0.02$ & $2.52 \pm 0.03$ \\
\hline Flux $^{\mathrm{a}}\left(10^{-9} \mathrm{erg} \mathrm{cm}^{-2} \mathrm{~s}^{-1}\right) \ldots \ldots \ldots \ldots \ldots \ldots \ldots \ldots$ & $5.9 \pm 0.3$ & $3.6 \pm 0.2$ & $10.2 \pm 0.5$ \\
\hline$\chi_{\nu}^{2}$ (d.o.f.) & $1.09(1936)$ & $0.99(1936)$ & $1.17(1936)$ \\
\hline
\end{tabular}

${ }^{\text {a }}$ Flux is evaluated in the $0.5-10 \mathrm{keV}$ range. 


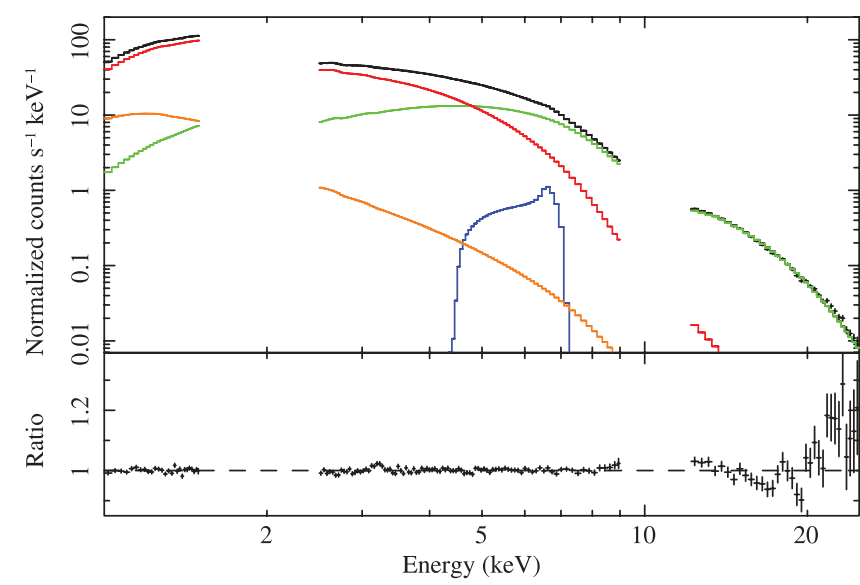

FIG. 1.-Suzaku spectrum of Serpens X-1. For clarity only the XIS2 (1$9 \mathrm{keV}$ ) and PIN (12-25 keV) data are plotted, and the XIS2 data have been rebinned. The black line shows the overall model; the colors distinguish the separate model components. Green is the blackbody, red is the multicolor disk blackbody, orange is the power law, and blue is the diskline.

For slowly rotating neutron stars, the gravitational potential around a neutron star is expected to be very close to a simple Schwarzschild potential. In fact, the neutron star spin for $4 \mathrm{U}$ 1820-30 and GX 349+2 inferred from the difference between the frequencies of the $\mathrm{kHz}$ QPOs is less than $300 \mathrm{~Hz}$, which would imply that the spin parameter $j \equiv c J / G M^{2}<0.3$ (Miller et al. 1998). Frame-dragging corrections for such spins should give errors of much less than 10\% (Miller et al. 1998). We therefore fit the iron line profiles with a model of line emission from a relativistic accretion disk assuming the Schwarzschild metric (the diskline model; Fabian et al. 1989). The line profiles in all objects can be well fit by this model. When fitting the lines we restrict the line energy to between 6.4 and $6.97 \mathrm{keV}$ (the allowed range for different ionization states of $\mathrm{Fe} \mathrm{K}$ emission). The inner radius, inclination, and disk emissivity are free parameters in the fit, while the outer disk radius is fixed at a large value $\left(1000 R_{G}\right)$.

The inner radius, $R_{\text {in }}$, of the accretion disk (in units of gravitational radii, $R_{G}=G M / c^{2}$ ) is important for shaping the line and is a parameter of the line fit. We are therefore able to measure the inner radius of the accretion disk in the three objects. The full diskline parameters are given in Table 3 . The inner radius of the accretion disk places an upper limit on the neutron star radius. In general, we find that these inner disk radii are in the range of $7 R_{G}-8 R_{G}$, which is equivalent to $14.5-16.5 \mathrm{~km}$ for a $1.4 M_{\odot}$ neutron star.

We checked that the diskline is the appropriate line model to use. Alternative models have been developed for emission lines formed in the spacetime around a spinning black hole. We find that fits with the "Laor" model (Laor 1991) for a maximally spinning black hole give inner disk radii that are consistent with the diskline fits. We chose the diskline model, since reasonable expectations for neutron star parameters suggest a spin parameter $<0.3$.

In addition to fitting with the diskline model, it is also possible to put a lower limit on the gravitational redshift, $z=G M / R c^{2}$, from fitting the asymmetric line profiles with two Gaussians, one narrow to fit the peak, and one broad to fit the red wing. We compare the measured center energy of the broad Gaussian component with the line rest energy. If the disk extends all the way down to the neutron star surface, one could naively interpret the energy shift as the surface redshift. Doing this we get redshifts in the range $0.1-0.2$, providing a consistency check with diskline fits.

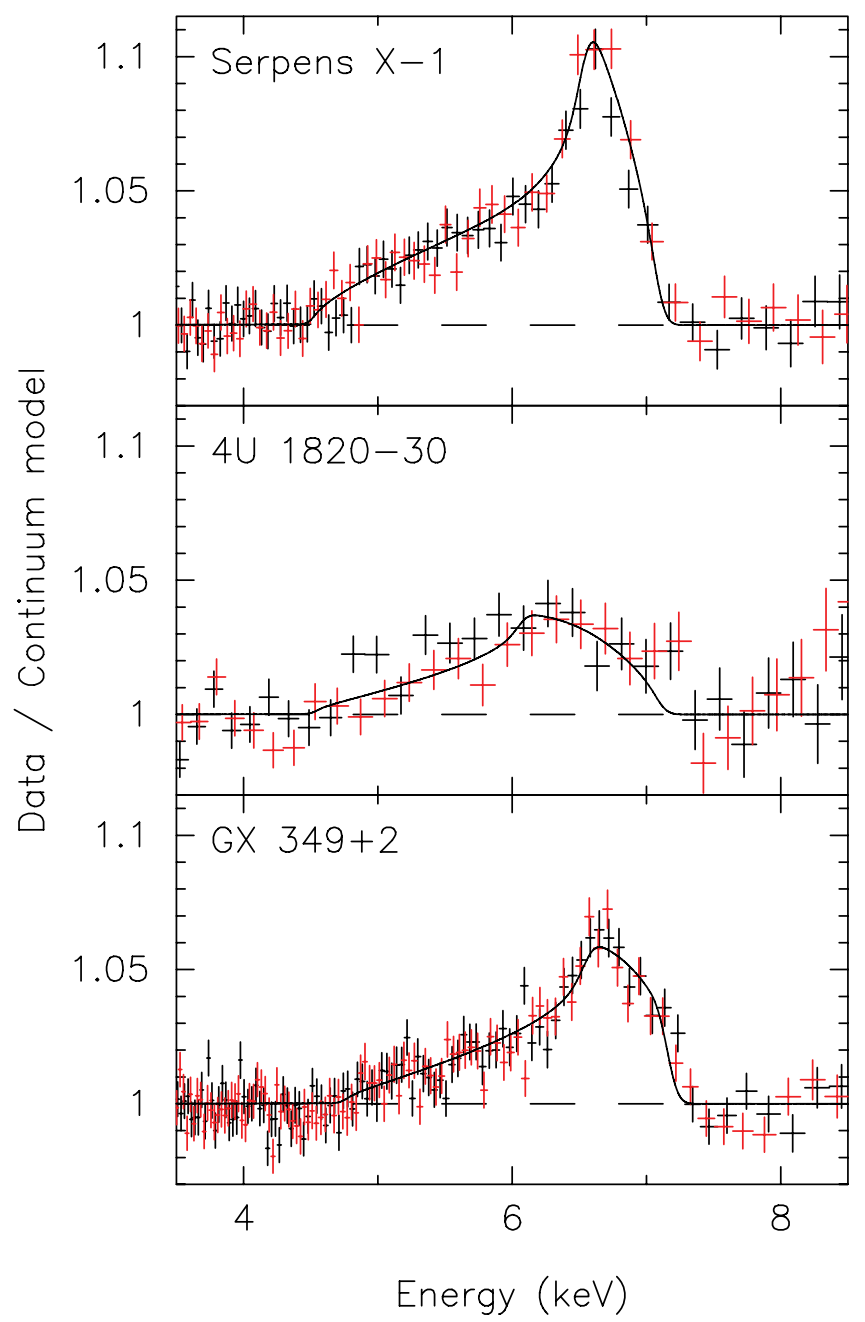

FIG. 2.- Iron lines in the three neutron star LMXBs observed with Suzaku. The ratio of count rate to continuum model is shown vs. energy. Black points are for data from the XIS 2 detector, and red points are from the XIS 3 detector. The solid line is the best-fitting diskline model.

We also note that there is expected to be a "boundary layer" between the inner accretion disk and the neutron star. This material is expected to be optically thick, and not in a Keplerian orbit around the star. The blackbody component of our continuum model can be interpreted as this component. In alternative continuum models this is often modeled as a Comptonized component, although the high effective optical depth means that it essentially behaves as a blackbody. In neutron star spectra there do appear to be two thermal components that follow $T^{4}$ as the source luminosity increases ( Lin et al. 2007), one a Keplerian accretion disk and the other a small, optically thick boundary layer. Moreover, the line profiles that we observe are well fit by the diskline model, suggesting that such a boundary layer does not strongly affect the line profile. In fact, the inner disk radii that we measure may imply that the boundary layer is small, for reasonable neutron star radii. In addition, the frequencies of $\mathrm{kHz}$ QPOs observed in neutron star systems are likely associated with the orbital frequency of the inner disk, and the frequency of this QPO is similar to the expected Keplerian frequency there (Miller et al. 1998), indicating that the inner disk is likely in Keplerian orbits.

Finally, we note that to achieve the asymmetric lines that are observed here the iron line emission must originate in the accretion disk. Alternative models for line broadening, such as 
TABLE 3

Diskline Parameters and kHz QPO Details

\begin{tabular}{|c|c|c|c|}
\hline Parameter & Serpens $X-1$ & $4 \mathrm{U} 1820-30$ & GX $349+2$ \\
\hline ine en & 6.8 & 6.97 & 6.97 \\
\hline in $(G \Lambda$ & 7.7 & $\begin{array}{l}0.10 \\
+1.4\end{array}$ & 0.4 \\
\hline Emissivity index, $\beta \ldots .$. & $-4.8_{-0.8}^{+0.4}$ & $-4.3_{-2.1}^{+1.1}$ & $-4.1 \pm 0.3$ \\
\hline Inclir & $26 \pm 2$ & $19_{-18}^{+5}$ & $=1$ \\
\hline $\left.0^{-3}\right)$ & 6.6 & 0.4 & 0.6 \\
\hline ) & $132=$ & $51=$ & $76 \pm 6$ \\
\hline$\chi_{\nu}^{2}($ d.o.f. $) \ldots$ & $1.05(3851)$ & $1.00(3851)$ & $1.06(3851)$ \\
\hline$R_{\text {in }}(\mathrm{km})$ from & $15.9 \pm 1.0$ & $13.8_{-1.4}^{+2.9}$ & $16.5 \pm 0.8$ \\
\hline Upper kHz QI & $\ldots$ & $1060 \pm 20$ & $978 \pm 9$ \\
\hline$R_{\mathrm{in}}(\mathrm{km})$ from $\mathrm{kHz} \mathrm{QPO} . . . \ldots . .$. & $\ldots$ & $16.1 \pm 0.2$ & $17.0 \pm 0.1$ \\
\hline
\end{tabular}

Notes.-The $R_{\text {in }}$ measurements given in $\mathrm{km}$ are evaluated at $M=1.4 M_{\odot}$. Ser X-1 does not have a detected $\mathrm{kHz}$ QPO. Kilohertz QPO measurements are taken from Zhang et al. (1998a) for 4U 1820-30, where we choose the value where the upper $\mathrm{kHz}$ QPO frequency reaches a maximum and remains constant with increasing count rate. For GX $349+2$ we use the $\mathrm{kHz}$ QPO measurement from Zhang et al. (1998b)

Comptonizing coronae, would require a very high optical depth, making the Comptonizing material effectively a disk (Reynolds \& Wilms 2000).

\section{DISCUSSION}

Suzaku observations of Ser X-1, 4U 1820-30, and GX 349+2 have revealed broad, relativistic $\mathrm{Fe} \mathrm{K}$ emission lines in each of the objects. By fitting with the diskline model, we were able to measure the inner radius of the accretion disk, and hence an upper limit on the neutron star radius. Only in one case previously has a clearly asymmetric line profile been observed (Bhattacharyya \& Strohmayer 2007), and that was in Ser X-1 with XMM-Newton. The iron line that we see in Ser X-1 with Suzaku is very similar in shape to that seen independently with $X M M$-Newton by Bhattacharyya \& Strohmayer (2007), and the inner disk radii are similar, although not quite consistent within $1 \sigma$. The observations presented here are more sensitive and have a broader continuum coverage, allowing tighter constraints on the inner disk radius. The fact that relativistic content is now clear in the independent work of Bhattacharyya \& Strohmayer (2007) and in the more sensitive Suzaku spectra presented here signals that these lines are robust and can now be used to constrain the nature of neutron stars.

In Figure 3 we show the constraints on radius and mass from these new Suzaku observations. They are completely independent of any previous constraints and rely on different physics. For comparison we include the constraints from $\mathrm{kHz}$ QPOs observed in X-ray binaries. We also show mass-radius curves from a variety of equations of state. The curves shown are not meant to be exhaustive but are just shown to demonstrate a range in massradius expected from different equations of state. While the radius upper limits from our current observations cannot rule out any equations of state, the potential of using these lines can be seen. Moreover, if masses for these neutron stars are determined, then the region of allowed mass-radius space is significantly reduced.

Indeed, our analysis of broad iron lines in two sources that exhibit kHz QPOs (4U 1820-30 and GX 349+2; Zhang et al. $1998 \mathrm{a}, 1998 \mathrm{~b}$ ) opens up a new way to estimate the masses of the neutron stars in these systems. The upper $\mathrm{kHz}$ QPO frequency is thought to be close to the orbital frequency at the inner edge of the optically thick emission (e.g., Miller et al. 1998); and, thus, from a radius close to the $R_{\text {in }}$ inferred from the iron line. In

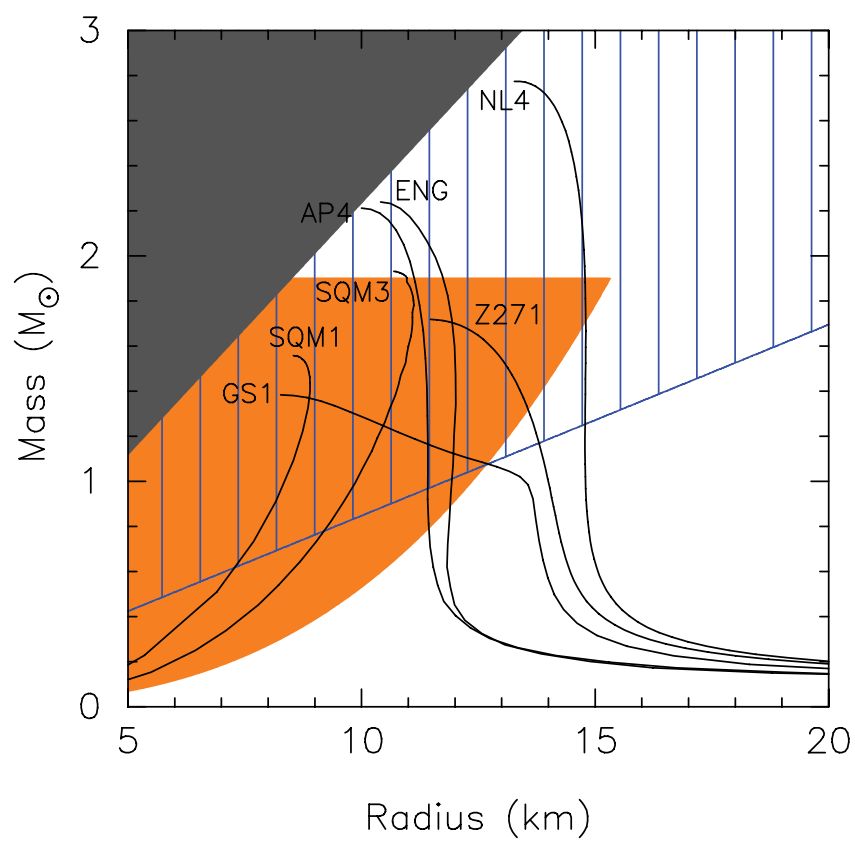

FIG. 3.-Neutron star gravitational mass and radius constraints. The gray region is ruled out because of causality (the sound speed must be less than the speed of light). The orange region is the allowed region from the highest frequency QPO observed (1330 kHz in 4U 0614+091; van Straaten et al. 2000) and assuming the neutron star spin, $c J / G M^{2}=0.2$, where $J$ is the stellar angular momentum. The blue region is allowed from this work on neutron star iron lines-here we plot the most well-constrained upper limit, which is from GX $349+2$. For comparison, we also plot the mass-radius curves for a variety of equations of state. All curves are as labeled in Lattimer \& Prakash (2001; see references therein for details of the equations of state), except for NL4, which is from Akmal et al. (1998), and Z271, which is from Horowitz \& Piekarewicz (2001).

Schwarzschild coordinates the expressions for orbital speed and frequency measured at infinity are the same as their Newtonian counterparts, $v_{\text {orb }}=(G M / r)^{1 / 2}$ and $\nu_{\text {orb }}=(1 / 2 \pi) \sqrt{G M / r^{3}}$. Therefore, a simultaneous measurement of speed and frequency allows a measurement of the stellar mass regardless of the radius:

$$
M=\frac{v_{\text {orb }}^{3}}{2 \pi G \nu_{\mathrm{orb}}} .
$$

Frame-dragging corrections to this expression for a spacetime with angular momentum parameter $j \equiv c J / G M^{2}$ are only of or$\operatorname{der} \sim j /(r / M)^{3 / 2}$ (e.g., Markovic 2000 ), which is less than $2 \%$ for the expected $j<0.3$. Simultaneous spectral and timing observations of $4 \mathrm{U} 1820-30$ would be especially valuable due to the tentative inference of a mass $M>2 M_{\odot}$ from a saturation of QPO frequency versus count rate and color (Zhang et al. 1998a; Bloser et al. 2000; see Méndez 2006 for a dissenting view).

Pending such a coordinated analysis, in Table 3 we present a simplified consistency test between the timing and spectral data in $4 \mathrm{U} 1820-30$ and GX 349+2. For a given QPO frequency, $\nu$, the radius is bounded from above by $R_{\max }=\left[G M /\left(4 \pi^{2} \nu^{2}\right)\right]^{1 / 2}$; hence, for a given source the largest observed frequency yields the smallest upper limit $R_{\max }$. We list these limits in Table 3, assuming a mass $M=1.4 M_{\odot}$. The general consistency between spectral and timing limits is encouraging. We also see that the best values for $4 \mathrm{U} 1820-30$ are somewhat offset, and find that the agreement would be improved for higher mass (e.g., $M=$ $2 M_{\odot}$ works), but the current broad errors on the iron line and lack of simultaneous timing measurements prevent us from drawing conclusions at this time. 
We note that we find low values $\left(<30^{\circ}\right)$ for the system inclinations in all cases. This may be a selection effect, as the observed objects were chosen based on strong lines seen in previous $A S C A$ data (Asai et al. 2000), and the strongest lines are present in systems with the lowest inclinations. Ser X-1 and GX 349+2 do not have previously well defined inclinations, although Ser X-1 is expected to be $<60^{\circ}$ as no energy-dependent X-ray dips or eclipses are seen (Frank et al. 1987) and GX 349+2 is also suggested to have a low inclination (Wachter \& Margon 1996; O'Neill et al. 2001). For $4 \mathrm{U} 1820-30$, an inclination of $35^{\circ}-50^{\circ}$ was determined from UV observations (Anderson et al. 1997), and observations of the X-ray superburst find limits that are consistent with this (Ballantyne \& Strohmayer 2004), although also allowing slightly lower inclinations. Differences in the inclination may be reconciled if the inner disk is at a different inclination to the binary system. This may be possible, as radio jets are not always seen to align with the binary inclination. For example, the radio jet in the black hole X-ray binary GRO J1655-40 (Hjellming \& Rupen 1995) is inclined at a different angle than the inclination binary system determined from optical light curves (Orosz \& Bailyn 1997; Greene et al. 2001).

The presence of relativistic iron lines in neutron stars, as well as in black holes, suggests that despite the physical and observational differences between the two types of objects, the accretion disks must be fairly similar. Extremely broad iron lines in some black hole sources are thought to be due to spinning black holes allowing the inner disk to extend farther in (e.g., Miller et al. 2004; Brenneman \& Reynolds 2006). The iron lines that we observe in these neutron stars strengthen this argument, as the neutron star iron lines all have inner disk radii much greater than these extreme cases in black holes.

Finally, in observations of iron lines in black holes, a strong reflection component, or "hump," is generally observed above $10 \mathrm{keV}$ (e.g., Miniutti et al. 2007). In our observations we do not find strong evidence for such a reflection component. Currently, reflection spectra have only been calculated for an ionizing spectrum that is power law in nature. We find, however, that neutron star spectra turn over and drop off faster than a power law, so any reflection hump would be less apparent.

\section{CONCLUSIONS}

We have found broad, relativistic Fe $\mathrm{K}$ emission lines in three neutron star LMXBs (Ser X-1, 4U 1820-30, and GX 349+2) using Suzaku. From diskline fits to the lines, we were able to measure the inner radius of the accretion disk, and hence place an upper limit on the neutron star radius. However, present measurements cannot currently rule out any reasonable equations of state.

We found that the inner disk radii that we measure with iron lines are consistent with the inner disk radii implied by $\mathrm{kHz}$ QPOs in $4 \mathrm{U} 1820-30$ and GX 349+2, supporting the inner disk nature of $\mathrm{kHz}$ QPOs. In addition the iron lines observed in these neutron stars are narrower than those in the black holes that are thought to be spinning, as one would expect if the spin interpretation is correct.

Recently, new observations are yielding important progress in understanding neutron stars, such as constraining the allowed properties of the crust (e.g., Cackett et al. 2006; Watts \& Strohmayer 2006; Strohmayer \& Brown 2002). However, determining the equation of state of matter in the core still remains a difficult problem. As currently one single method will not conclusively determine the equation of state, it is critical to have multiple, independent methods to measure masses and radii of neutron stars. The advantage of using Fe $\mathrm{K}$ emission lines as a probe of the neutron star radius is that they only require short observations to clearly reveal the relativistic lines, and they do not require any knowledge of the distance to the object. In addition, there are likely to be many neutron star low-mass X-ray binaries that have these lines (e.g., Asai et al. 2000). Thus, using relativistic iron lines in neutron stars offers a promising, complementary method to constrain neutron star radii. Future proposed X-ray observatories such as Constellation- $X$ and XEUS will be able to place extremely tight limits on even weak lines.

We thank A. Steiner and J. Lattimer for very kindly providing the mass-radius curves for various equations of state. J. M. M. acknowledges funding from NASA through the Suzaku GO program.

\section{REFERENCES}

Abramowicz, M. A., Bulik, T., Bursa, M., \& Kluźniak, W. 2003, A\&A, 404, L21

Akmal, A., Pandharipande, V. R., \& Ravenhall, D. G. 1998, Phys. Rev. C, 58, 1804

Anderson, S. F., Margon, B., Deutsch, E. W., Downes, R. A., \& Allen, R. G. 1997, ApJ, 482, L69

Arnaud, K. A. 1996, in ASP Conf. Ser. 101, Astronomical Data Analysis and Systems V, ed. G. Jacoby \& J. Barnes (San Francisco: ASP), 17

Asai, K., Dotani, T., Nagase, F., \& Mitsuda, K. 2000, ApJS, 131, 571

Ballantyne, D. R., \& Strohmayer, T. E. 2004, ApJ, 602, L105

Bhattacharyya, S., \& Strohmayer, T. E. 2007, ApJ, 664, L103

Bhattacharyya, S., Strohmayer, T. E., Miller, M. C., \& Markwardt, C. B. 2005, ApJ, 619, 483

Bloser, P. F., Grindlay, J. E., Kaaret, P., Zhang, W., Smale, A. P., \& Barret, D. 2000, ApJ, 524, 1000

Bogdanov, S., Rybicki, G. B., \& Grindlay, J. E. 2007, ApJ, 670, 668

Brenneman, L. W., \& Reynolds, C. S. 2006, ApJ, 652, 1028

Cackett, E. M., Wijnands, R., Linares, M., Miller, J. M., Homan, J., \& Lewin, W. H. G. 2006, MNRAS, 372, 479

Cottam, J., Paerels, F., \& Mendez, M. 2002, Nature, 420, 51

Di Salvo, T., Iaria, R., Méndez, M., Burderi, L., Lavagetto, G., Robba, N. R., Stella, L., \& van der Klis, M. 2005, ApJ, 623, L121

Fabian, A. C., Rees, M. J., Stella, L., \& White, N. E. 1989, MNRAS, 238, 729

Frank, J., King, A. R., \& Lasota, J.-P. 1987, A\&A, 178, 137

Greene, J., Bailyn, C. D., \& Orosz, J. A. 2001, ApJ, 554, 1290

Heinke, C. O., Rybicki, G. B., Narayan, R., \& Grindlay, J. E. 2006, ApJ, 644, 1090
Hirano, T., Hayakawa, S., Nagase, F., Masai, K., \& Mitsuda, K. 1987, PASJ, 39,619

Hjellming, R. M., \& Rupen, M. P. 1995, Nature, 375, 464

Ho, W. C. G., Kaplan, D. L., Chang, P., van Adelsberg, M., \& Potekhin, A. Y. 2007, MNRAS, 375, 821

Horowitz, C. J., \& Piekarewicz, J. 2001, Phys. Rev. Lett., 86, 5647

Klähn, T., et al. 2006, Phys. Rev. C, 74, 035802

Kong, A. K. H., Miller, J. M., Mendez, M., Cottam, J., Lewin, W. H. G.,

Paerels, F., Kuulkers, E., \& Wijnands, R. 2007, ApJ, 670, L17

Lamb, F. K., \& Miller, M. C. 2001, ApJ, 554, 1210

Laor, A. 1991, ApJ, 376, 90

Lattimer, J. M., \& Prakash, M. 2001, ApJ, 550, 426 2007, Phys. Rep., 442, 109

Lin, D., Remillard, R. A., \& Homan, J. 2007, ApJ, 667, 1073

Marković, D. 2000, preprint (astro-ph/0009450)

Méndez, M. 2006, MNRAS, 371, 1925

Miller, J. M. 2007, ARA\&A, 45, 441

Miller, J. M., et al. 2004, ApJ, 606, L131

Miller, M. C., Lamb, F. K., \& Psaltis, D. 1998, ApJ, 508, 791

Miniutti, G., et al. 2007, PASJ, 59, 315

Mitsuda, K., et al. 2007, PASJ, 59, 1

Nandra, K., George, I. M., Mushotzky, R. F., Turner, T. J., \& Yaqoob, T. 1997, ApJ, 477, 602

O’Neill, P. M., Kuulkers, E., Sood, R. K., \& Dotani, T. 2001, A\&A, 370, 479 Oosterbroek, T., Barret, D., Guainazzi, M., \& Ford, E. C. 2001, A\&A, 366, 138 Orosz, J. A., \& Bailyn, C. D. 1997, ApJ, 477, 876

Reynolds, C. S. 1997, MNRAS, 286, 513 
Reynolds, C. S., \& Wilms, J. 2000, ApJ, 533, 821

Stella, L., Vietri, M., \& Morsink, S. M. 1999, ApJ, 524, L63

Strohmayer, T. E., \& Brown, E. F. 2002, ApJ, 566, 1045

Titarchuk, L. 2002, ApJ, 578, L71

van Paradijs, J., \& Lewin, W. H. G. 1987, A\&A, 172, L20

van Straaten, S., Ford, E. C., van der Klis, M., Méndez, M., \& Kaaret, P. 2000, ApJ, 540, 1049

Wachter, S., \& Margon, B. 1996, AJ, 112, 2684

Watts, A. L., \& Strohmayer, T. E. 2006, ApJ, 637, L117
White, N. E., Peacock, A., Hasinger, G., Mason, K. O., Manzo, G., Taylor, B. G., \& Branduardi-Raymont, G. 1986, MNRAS, 218, 129

White, N. E., Peacock, A., \& Taylor, B. G. 1985, ApJ, 296, 475

Zavlin, V. E. 2008, in The 363rd Heraeus Seminar on Neutron Stars and Pulsars, ed. W. Becker, H. Lesh, \& J. Trümper (Berlin: Springer), in press (astro-ph/0702426)

Zhang, W., Smale, A. P., Strohmayer, T. E., \& Swank, J. H. 1998a, ApJ, 500, L171

Zhang, W., Strohmayer, T. E., \& Swank, J. H. 1998b, ApJ, 500, L167 